\title{
Quantitative investigation of Nippostrongylus brasiliensis acting as vector of Trypanosoma congolense or Trypanosoma brucei in rat.
}

\author{
by J. O. SIMAREN
}

[Department of Zoology, University of Ife, Nigeria]

\begin{abstract}
Résumé
Devant la faculté, pour certains Helminthes parasites, de transmettre divers agents pathogènes à de nouveaux hôtes, l'auteur a recherché si le Nématode du rat, Nippostrongylus brasiliensis ne pourrait transmettre expérimentalement à d'autres rats Trypanosoma congolense ou Trypanosoma brucei.

Un premier lot de rats a été simultanément infesté par $T$. congolense et $N$. brasiliensis; un deuxième lot par $T$. brucei et $N$. brasiliensis; un troisième lot a été infesté par $T$. congolense et ultérieurement par $N$. brasiliensis; enfin un quatrième lot était infesté d'abord par $T$. brucei et ensuite par $N$. brasiliensis.

Les larves infestantes du troisième stade, obtenues en culture à partir des fécès de chacun des lots doublement infestés, ont été inoculées à des rats sains. L'examen répété du sang n'a jamais révélé la présence de Trypanosomes transmis par ces larves.
\end{abstract}

\section{Summary}

The ability of the rat Nematode Nippostrongylus brasiliensis to transmit $T$. congolense or $T$. brucei experimentally was investigated.

One group of rats was infected simultaneously with $T$. congolense and $N$. brasiliensis and another group with $T$. brucei and $N$. brasiliensis. A third group of rats was infected with $T$. congolense and then later with $N$. brasiliensis, while a fourth group was infected with $T$. brucei and then with $N$. brasiliensis. Infective third stage larvae cultured from the feces of each doubly infected groups were injected into healthy rats. Blood from the recipient hosts was checked repeatedly to see if the Trypanosomes had been transmitted by $N$. brasiliensis. The results were negative. 
It seems however that under the condition of these experimental protocols, if transmission of the Trypanosomes by $N$. brasiliensis occurred, the rate must have been very low. In conclusion the results showed that no Trypanosomes were present in the tissues or digestive tract of any of the developing stages of the Nematode; either similarly was there any worm revealing Trypanosomes of a significant nature in the blood or found in such in Vitro.

\section{Introduction.}

Literature has revealed that not only ectoparasites such as mosquitoes, ticks act as vector and reservoir of parasitic organisms but certain helminths have also been proved involved propagation and transmission of disease agents. Eggs and larval stages of Heterakis gallinae transmit Histomonas meleagridis in chicken (Graybill et al. 1920). Lungworm acts as intermediate host for the Virus swine influenza (Shope 1939, 1941, 1943). Phillip (1955) demonstrated that the fluke Nanophetus salminocal acted as a vector and intermediate host for Neorickettsia he!minthoeca, a fatal intracytoplasmic and rickettsia like disease agent. Trichinella spiralis according to Syverton et al. (1947) transmitted the virus lymphocytic chorriomeningitis.

With this knowledge this investigation was therefore designed to test the possibility of any transmission of hemoflegellate Trypanosome congolense or T. brucei experimentally in induced rat by the rat Nematode Nippostrongylus brasiliensis.

\section{Materials and Methods.}

Male and female white laboratory bred rats which were protozoan and helminth free were used as host in the investigation. The test agents were the hemoflagellates Trypanosoma brucei and Trypanosoma congo'ense, and the nematode Nippostrongylus brasiliensis. By weekly passage of separate stock of the trypanosomes and bi-weekly transfer of Nippostrongylus into healthy rats, these organisms are being continuously maintained in our laboratory. Few drops of blood taken from tail of rat infected with each trypanosome species were diluted with $5 \mathrm{ml}$ of 0,85 per cent of Sodium Chloride depending on the density of the trypanosomes. The suspension was mixed and 1,600 trypanosomes of each species quantitatively determined were inoculated subcutaneously and intraperitoneally into rats.

The presence and degree of trypanosomes was checked daily (a) by examining a wet mount of blood drops from the tail on slides and $(b)$ by examining stained blood smears.

The culture method for Nippostrongylus brasiliensis was essentially the same as that of Yokogawa (1922). Isolation and preparation of larvae from culture for injection was by a slight modification of Baerman apparatus (Belding 1952). Larvae isolated were washed at least 4 times in 0,85 per cent Sodium chloride solution, centrifuged at low speed and supernatant removed. The centrifuged tube of larvae was 
agitated and 0.1 to $0.3 \mathrm{ml}$ of sample withdrawn and counted on a disecting binocular microscope. An average of six samples was made and the amount of solution adjusted to yield the number of larvae needed for infection. All rats were infected with 2.000 Nippostrongylus larvae regardless of the routes of infection.

$2,3,6,7,8$ and 9 days after infection, groups of infected rats were sacrificed for worm counts by slit-opening the small intestine cut into small pieces of 0,85 per cent Sodium Chloride. Egg collection were examined by adaptation of Lane's Direct Centrifugation flotation method. Heavily infected lungs were washed in 0,85 per cent Sodium Chloride and minced with a sharp-razor blade. The larvae were released quickly by placing the minzed lungs in warm saline using Baermann Apparatus. The collected larvae were examined microscopically on slides and then stained with Giemsa.

\section{Experiments and Observations.}

A set of three experiments were conducted. The first one was to test the possible transmission of $T$. congolense or T. brucei by $N$. brasiliensis when all the two organisms were simultaneously injected.

EXPERIMENT I. - Group A of ten rats 7 weeks old were given $2.000 \mathrm{~N}$. brasiliensis subcutaneously, and simultaneously saline suspension of $800 \mathrm{~T}$. brucei subcutaneously and $800 \mathrm{~T}$. brucei intraperitoneally. Group B of ten rats were similarly and quantitatively injected with $N$. brasiliensis and $T$. congolense. Eight rats were kept as control of which, two were injected $2.000 \mathrm{~N}$. brasiliensis only; two with $T$. brucei alone, two with $T$. congolense and the other two was kept for observation and comparing the general conditions of the rats. The course of $N$. brasiliensis infection was checked daily by fecal smears. Eggs appeared in the feces of both doubly and singly infected animals. No egg counts were made since the investigation was not objectively quantitative. Fresh blood smears and blood stained smears with Giemsa made daily confirmed the present of trypanosomes in the blood. T. congo!ense infections reached their peak of multiplication about the 8 th day after infection while $T$. brucei around the 10th day when death set in. General debility and loss of appetite were exhibited by doubly infected rats but less pronounced in rats receiving trypanosomes alone.

Attempts were made to determine if the larvae of $N$. brasiliensis developing in feces from doubly infected rats could transmit $T$. brucei or $T$. congolense to healthy rats.

A dosage of 2.000 larvae was injected subcutaneously into 12 healthy rats. A dosage of 2.000 larvae prepared from rats infected with $N$. brasiliensis alone was also injected into 3 rats which serve as controls. Three healthy rats were injected with $0.3 \mathrm{ml}$. saline suspension of $T$. brucei intraperitoneally and subcutaneously. Three other healthy rats were similarly injected with $T$. congolense. No trypanosomes were found in the blood of the rats inoculated with larvae cultured from the feces 
from doubly infected rats. All blood examined were similar. At necropsy small intestines were examined for the presence of adult worms.

EXPERIMENT II. - Was performed to test possible transmission of $T$. congolense by $N$. brasiliensis when the two agents were given at different times. Twelve rats seven weeks old were injected with 1,600 saline suspension of $T$. congolense intraperiotoneally and subcutaneously as in experiment I. Two of these rats one day after initial injection of $T$. congolense, two at 2 days and two at 3 days respectively, were inoculated with 2,000 Nippostrongylus larvae. On the sixth day when large numbers of the trypanosomes appeared in the blood, 2,000 Nippostrongylus larvae were inoculated into each of remaining six rats. Twelve other rats were similarly challenged with T. brucei and Nippostrongylus.

An inoculum of 1,000 larvae recovered prepared from each of the cultures mane from the feces of each of the twelve infected rats was injected to twelve healthy rats. Daily examination of fresh blood and stained smear revealed no trypanosomes. Consequently, the time variation adopted between the administration of the two test agents in each case has no significant effect. Findings from these two experiments $s^{\circ} \mathrm{m}$ rather inconclusive and since Taliaferro et al. (1939) assumed that $N$. brasiliensis feeds on blood, it was thought that it might be possible for $T$. congolense or $T$. brucei to be ingested by adult $N$. brasiliensis during the larval life cycle in the host.

Further test search were made for trypanosomes in Nippostrongylus larvae, larval lung-stages, early intestinal larval stages, and adult worms recovered from doubly infected rats.

Many adult male and female worms obtained from doubly infected rats of experiment I were examined under binocular disecting microscope for trypanosomes and for signs of internal motility. No movements other than peristaltic motion of the intestinal contents was observed.

By means of microdissection of the adult recovered worms, unstained alimentary canal was examined under oil immersion for signs of trypanosomal movement. A modification of Giemsa's stain was used in attempt to detect erythrocytes in the worm's intestine. A few drops of concentrated Giemsa stain was placed under the cover slip and the slide was left for a few hours to allow the solution to spread throughout the intestinal tissue. The covership was sealed with vaseline and the preparation examined under oil immersion. No trypanosomes or erythrocytes were detected.

Group C of 4 rats were given 1,600 T. brucei intraperitoneally, while group D of 4 rats were given 1,600 T. congolense intraperitoneally. Trypanosomes were abundant in the blood on the 6th and 7th day respectively, at which time all rats were challenged with $2,000 \mathrm{~N}$. brasiliensis larvae. Two rats from each group $\mathrm{C}$ and D were sacrificed 24 hours later. Their lungs were removed and lung stage larvae isolated were also examined for motile trypanosomes but none. Hemorrhagic areas of the lungs were teased between two glass slides and larvae were found trapped in the lungs, but no trypanosomes. Rats left in groups 
C and D were necropsied 48 hours thereafter and their lungs and intestine removed. Examination of the worms found in anterior part of the intestine were slightly different from the lung stage worms in appearance. Intestinal walls and the cuticule were pinkish and dark. Whole stained worms yielded no trypanosomes or erythrocytes.

A third experiment on the feeding habits of $N$. brasiliensis was performed to determine whether trypanosomes or erythrocytes might be ingested by the worm in the in Vitro. Blood heavy with trypanosomes was obtained from infected rats. One milliliter of a 2 per cent citrate solution (1 gm citrate $/ 50 \mathrm{ml}$ Ringer's solution) was added to $2 \mathrm{ml}$ of blood to prevent clotting. Infective larvae obtained from charcoal fecal culture were washed many times in sterile 0,85 per cent $\mathrm{NaCl}$ and sterilized for 30 minutes in a 0,1 per cent mercuri chloride solution to rid the larvae of bacterial contamination. Lung stage larvae and early intestinal stages were also obtained as described earlier. The in Vitro test consisted of a ring of sterile vaseline on a $1 \times 3$ inch slide. After 1-2 drops of prepared blood was placed inside the ring, 20 worms suspended in saline were added. A cover slip was gently placed over the vaseline without any inhibition to observation of worm movement. The nematodes were observed to take in fluid but no erythrocytes or trypanosomes. Thirty worms of various stages were stained with Giemsa. Microscopic observation revealed concentration of fat globules and brownish pigment granules. No trypanosomes or erythrocytes were found in the worms.

\section{Results and Discussion.}

The operating hypothesis was that if $N$. brasiliensis feed on blood as it is reported and the blood contains $T$. congolense and $T$. brucei in this investigation, then the nematode might ingest the trypanosomes and might act as an intermediate host transmitting the trypanosomes. It seems possible since some literature findings concerning diseases have incriminated helminths as reservoirs and vectors of disease organism. Black head of turkeys caused by a protozoan, swine influenza by a virus and salmon poisoning of dogs caused by a rickettsia have been proved to be transmitted by helminth intervention.

Cyclical development of $N$. brasiliensis and the trypanosomes indicated that parasitemia of these organisms reached a peak by the end of first week after infection, hence the injection of both organisms simultaneously in order to facilitate the greatest possible contact between them. Under the conditions of this investigation, the preliminary studies and experiment $\mathrm{I}$ indicated that $N$. brasiliensis did not transmit $T$. congolense or $T$. brucei to a new experimental host when simultaneously administered.

It must not be overlooked that many natural factors might possibly affect the vector mechanism. Therefore time intervals was allowed between injection of the test - agents in experiment II. The intervals play no significant role in facilitating transmission of the trypanosome infection. The results from this experiment again indicated that $N$. brasiliensis did not act as a vector of either $T$. congolense or $T$. brucei. Stein et al. (1939) studied the role of nematode endoparasites in the transmission of 
infectious anemia through a series of experiments on horses using a saline extract of washed and macerated bodies of worms genus Strongylus obtained from horses with infectious anemia. They found that when extract of third stage larvae of Strongylus harboured by infected horses was injected into normal horses, no diseases was observed, they concluded that the larvae do not contain the virus and the infectious anemia is not transmitted by Strongylus. This investigation is somehow parallel. Since the first two experiments showed negative results, perhaps it may be that the internal environment of the nematode is antagonistic to the retention and multiplication of the protozoan but one cannot rule out the possibility that the trypanosomes might be ingested by $N$. brasiliensis during the latter's feeding stages.

Additional search was made for the trypanosomes or erythrocytes inside the worms. Microscopic examination of whole adult worms, dissected and crushed-stained mount of the alimentary canal gave no positive presence of trypanosomes except dark and brownish erthrocyte-like particles.

Since no trypanosomes were found in the adult stages of the worms, further attempts were made to demonstrate the presence of the trypanosomes in the lung and early intestinal stages of $N$. brasiliensis. Yokogawa (1922) and Twohy $(1955,1956)$ reported that the period of growth of $N$. brasiliensis carried via the blood to the lung (where they remain) is between 19-50 hours after infection. With observations of this fact, the lungs showed slight hemorrhages along the routes of migration of the larvae. However no trypanosomes or erythrocytes were found in all recovered worms examined. Digestive tracts of some larvae showed presence of pigment-like particles. Moderate tissue damage were seen in the intestine and occasionally within it were found colonies of worms in fecal material tinged with blood. Tyzzer in 1934 identified Histomonas in the gut of $H$. gallinae and since then the only report of a protozoan within a worm was in 1959 when Kendall demonstrated Histomonas in the larvae of Heterakis. The fact that trypanosomes could not be demonstrated by any of the stages of $N$. brasiliensis in this studies is not surprising. The situation here is similar to that of black head in turkey, and Kendall's finding throw a supporting light on what happened in the present investigation.

\section{Bibliographie}

Belding (D. L.), 1952. - Clinical Parasitology, Second édition, Appleton-Century Craft Inc., New-York.

Graybill (H. W.) and Smith (T.), 1920. - Production of fatal blackhead in turkeys by feeding embryonated eggs of Heterakis papillosa. J. of Exptl. med., 31, 647-655.

Kendall (S. B.), 1959. - The Occurrence of Histomonas Indeagridis in Heterakis gallinae. Parasitol., 49, 169-172.

SHOPE (R. E.), 1939. - An intermediate host from the swine influenza virus, Science, 89, 441-442. 
-, 1941 a. - The swine Lungworm as reservoir and intermediate host for swine influenza virus 1. The presence of swine influenza virus in healthy and susceptible pigs. J. Exper. Med., 74, 41-48.

-, $1941 \mathrm{~b}$. - The swine Lungworm as reservoir and intermediate host for swine influenza virus 2. The transmission of swine lungworm. J. Exper. Med., 74, 49-61.

-, $1943 a$. - The swine Lungworm as reservoir and intermediate host for swine influenza virus 3. Factors influencing transmission of the virus and provocation of influenza. J. Exper. Med, 77, 111-126.

- $1943 \mathrm{~b}$. - The swine Lungworm as reservoir and intermediate host for swine influenza virus 4. The demonstration of masked swine influenza virus in lungworm larvae and swine under natural conditions. J. Exper. Med., 77, 127.

Stein (C. D.), Lucker (J. T.), Osteen (O. L.) and Gochernous (W. S.), 1939. - Studies on the possible role of endoparasites in the transmission of infectious anemia. J. Amer Vet. Assoc., 95, 536-541.

Syverton (J. T.) McCoy (O. R.), and KoOMEN (J.), Jr., 1947. - The transmission of the virus of lymphocytic choriomeningitis by Trichinella spiralis. J. Exper. Med., 85, 759-769.

TAfiaferro (W. H) and Sarles (M. P.), 1939. - The Cellular reactions in the skin, lungs and intestine of normal and immune rats after infection with Nippostrongylus muris. J. Inf. Dis., 64, 157-192.

TwoHY (D. W.), 1955. - The early migration and growth of Nippostrongylus muris in the rat. J. Parasitol., 41 (Suppl.), 49-50.

-, 1956. - The early migration and growth of Nippostrongylus muris in the rat. Amer. J. Hyg., 63, 165-185.

TYZZER (E. E.), 1934. - Studies on histomonasis or \& Blackhead \# infection in the chicken and turkey. Proc. Acad. Arts + Sciences, 69, 89.

YoKogAwA (S.), 1922. - The development of Heligmosomum muris yokogawai, a nematode, from the intestine of the wild rat. Parasitol., 14, 127-166. 